\title{
EL IMPACTO DE LA CRISIS EN LA CONCILIACIÓN DE LOS TIEMPOS COTIDIANOS Análisis de las familias del alumnado de Educación Primaria en Galicia
}

\author{
Rubén Martínez García \\ Universidad de Santiago de Compostela, Galicia, España
}

Ángela L. de Valenzuela

Universidad de Santiago de Compostela, Galicia, España

Laura Varela Crespo

Universidad de Santiago de Compostela, Galicia, España

Resumen El artículo se centra en estudiar la percepción de las familias gallegas con hijos e hijas escolarizados en Educación Primaria (6-12 años) sobre las repercusiones de la crisis económica en la conciliación de su vida familiar, laboral y personal, analizando su grado de satisfacción en relación a la misma. A partir de los datos del cuestionario elaborado ad hoc para las familias, los resultados indican que algo más de la mitad de las personas encuestadas ( $\mathrm{n}=$ 2037) consideran que la crisis no afectó en la armonización de sus tiempos cotidianos. Sin embargo, la mayoría no se sienten satisfechas con la organización de los tiempos de su vida diaria, evidenciándose que la crisis ha podido mermar su capacidad para alcanzar sus metas profesionales, familiares y personales.

Palabras-clave: crisis económica, conciliación, familia, satisfacción.

Resumo $\mathrm{O}$ artigo estuda a perceção das famílias galegas com crianças escolarizadas, a frequentar a Educação Primária (6-12 anos), sobre as repercussões da crise económica na conciliação da sua vida familiar, laboral e pessoal, analisando o seu grau de satisfação em relação à mesma. A partir dos dados do inquérito elaborado ad hoc para as famílias, os resultados indicam que mais de metade das pessoas entrevistadas $(\mathrm{n}=2037)$ consideram que a crise não afetou a harmonização dos seus tempos diários. Porém, a maioria não se sente satisfeita com a organização dos tempos da vida diária, comprovando-se que a crise tem diminuído a sua capacidade para alcançar as metas profissionais, familiares e pessoais.

Palavras chave: crise económica, conciliação, família, satisfação.

Abstract The paper focuses on studying the perception of Galician families with children enrolled in primary school (6-12 years old) about the impact of the economic crisis in reconciling their family, work and personal life, analyzing their satisfaction in regard to it. Based on data questionnaire prepared ad hoc for the families, the results indicate that more than half of the subjects $(n=2037)$ consider that the crisis did not affect the harmonization of their daily time. However, most of them are not satisfied with the organization of the times of their daily life, showing that the crisis could undermine their ability to achieve their professional, family and personal goals.

Keywords: economic recession, work-life balance, family, satisfaction.

Résumé L'article se concentre sur l'étude de la perception des familles galiciennes avec des enfants inscrits à l'école primaire (6-12 ans) de l'impact de la crise économique sur la conciliation entre travail, famille et vie personnelle, et l'analyse de leur satisfaction par rapport à celle-ci. D'après les données du questionnaire préparé ad hoc pour les familles, plus de la moitié des répondants $(n=2037)$ estiment que la crise n'a pas affecté l'harmonisation de leur temps quotidien. Cependant, la plupart ne sont pas satisfaits de l'organisation du temps de leur vie quotidienne, ce qui montre que la crise a pu nuire à leur capacité à atteindre leurs objectifs de carrière, familiaux et personnels.

Mots-clés: crise économique, conciliation, famille, satisfaction. 


\section{Introducción}

A menudo, en el imaginario social, la conciliación se circunscribe a las dificultades con las que se encuentran las madres para compatibilizar su horario laboral con las tareas de cuidado, una función tradicionalmente femenina limitada a la esfera doméstica. Desde hace ya varias décadas, la conciliación ha ido adquiriendo una especial relevancia en nuestra sociedad, resultando fundamental que las políticas públicas faciliten el acceso de la ciudadanía a las mejores estrategias para que todas las personas tengan el derecho a desarrollar una vida plena, en un marco de igualdad de oportunidades. En este sentido, trascender la concepción de la conciliación como un "problema de las mujeres" exige ineludiblemente la puesta en marcha de políticas familiares promotoras de una mayor corresponsabilidad.

En función de la perspectiva que se tome como referencia - individual, social o laboral -, son múltiples las acepciones que se pueden diferenciar respecto al término “conciliación" (Borràs, Torns y Moreno, 2007). Desde la dimensión individual, se entiende la conciliación como un problema personal, de modo que es la persona quien debe mejorar individualmente la organización de sus tiempos; la dimensión social, implica considerar los cambios acaecidos que afectan a la conciliación de los tiempos como una responsabilidad del conjunto de la sociedad; y desde la perspectiva laboral, se concibe como un aspecto que debe abordarse mediante los convenios colectivos circunscritos a la jornada laboral y al salario.

En la actualidad, el Estado de bienestar español, que comparte características esenciales con el resto de países del sur de Europa (Portugal, Grecia, Italia) -, entre ellas el importante papel asignado a las familias (en concreto, a las mujeres) en la garantía del bienestar y de la calidad de vida - , se encuentra en una situación de claro retroceso. No solo debido al significado que ha adquirido en la vida cotidiana de las familias la disminución de la inversión en el sistema público de los servicios sociales, con la consiguiente desaparición de recursos y prestaciones que garantizaban un nivel de vida digno y un mayor equilibrio entre las responsabilidades laborales y las tareas de cuidado, sino también porque determinadas conquistas sociales que parecían irreversibles están siendo intensamente cuestionadas (el derecho a un trabajo que permita hacer frente a los gastos básicos, a una vivienda digna, al disfrute del tiempo de ocio, etc.).

La regresión experimentada con las políticas de "austeridad" adoptadas en el año 2008 en respuesta a la crisis económica ha ido perfilando un Estado de bienestar "débil" (Antón, 2012), que cumple funciones mínimas de protección y garantía de la cohesión social. Una situación que exacerba los conflictos entre las obligaciones laborales y las responsabilidades derivadas de la cotidianeidad familiar (Andrade y Landero, 2015) e incrementa el malestar entre los trabajadores y trabajadoras, quienes experimentan una continua sensación de presión (Burke, 2009) que afecta a su bienestar personal (Baxter y Chesters, 2011) y a la calidad en el desempeño de las funciones profesionales (Sánchez-Vidal, Cegarra-Leiva y Cegarra-Navarro, 2011).

Dichas transformaciones son evidentes en las condiciones de vida de los hogares españoles, tanto por los propios cambios acaecidos en las estructuras 
familiares, como por otros factores - económicos, laborales, etc. - que influyen en las oportunidades de las familias para alcanzar su bienestar. Cabe afirmar que la incidencia de la crisis ha puesto de manifiesto una serie de aspectos que alertan del fracaso de las políticas familiares (Castro y Seiz, 2014): el aumento de la precarización en las condiciones de vida; la mayor incidencia de la exclusión social en las familias monoparentales (encabezadas por mujeres); la presencia de menores en el hogar - duplicándose las posibilidades de exclusión en el caso de que una mujer sea la sustentadora principal; las mayores dificultades de conciliación entre el trabajo y la familia; entre otras.

A finales de la década de los 90 en la Unión Europea, y posteriormente en los Estados miembros, se incorporaron en las políticas de empleo acciones orientadas a la conciliación de la vida familiar y laboral, en respuesta a la necesidad de la igualdad de oportunidades entre mujeres y hombres. Una línea estratégica que, todavía hoy en día, necesita ser impulsada, y que - en el caso español - forma parte de las prioridades del Plan Integral de Apoyo a la Familia (2015-2017), el cual articula un conjunto de medidas vinculadas a: el mercado de trabajo y la vida laboral; los servicios de cuidado y atención; así como la formación, información y sensibilización sobre esta temática. A nivel normativo-legal, se identifican en España dos leyes fundamentales: la Ley 39/1999, de 5 de noviembre, para promover la conciliación de la vida familiar y laboral de las personas trabajadoras, y la Ley Orgánica 3/2007, de 22 de marzo, para la igualdad efectiva de mujeres y hombres.

En la Comunidad Autónoma de Galicia, ámbito territorial del presente estudio, destaca la Ley $7 / 2004$, de 16 de julio, gallega para la igualdad de mujeres y hombres, la cual - bajo el principio de transversalidad en la aplicación de la igualdad de oportunidades en todas las políticas y acciones competencia de la Xunta de Galicia - establece como criterio general de actuación la conciliación del empleo y de la vida familiar de las mujeres y hombres y el fomento de la individualización de los derechos tendentes a esta conciliación. Asimismo, la Ley 2/2007, de 28 de marzo, del trabajo en igualdad de las mujeres de Galicia incide en la necesidad de la inserción laboral de las mujeres como uno de los pilares esenciales para hacer efectivo el principio de igualdad de oportunidades, al constituir el ámbito laboral uno de los escenarios en el que la desigualdad es más visible.

Sin embargo, aun considerando estos avances, el apoyo público para que mujeres y hombres puedan conciliar la vida laboral y las responsabilidades familiares resulta insuficiente, especialmente si se centra el foco en el impacto real de las políticas públicas en la vida cotidiana de las familias españolas. Según la encuesta Opiniones y Actitudes sobre la Familia (2014) elaborada por el Centro de Investigaciones Sociológicas (CIS), el impacto de la crisis económica en las familias desde que comenzó el año 2008 se manifiesta fundamentalmente en el ámbito laboral un $38,6 \%$ de las personas entrevistadas indican que han empeorado sus condiciones de trabajo, frente a un 3,7\% que percibe una mejora -, incidiendo el $48,5 \%$ de las personas participantes en la necesidad de flexibilizar la jornada laboral de trabajadores y trabajadoras con responsabilidades familiares como una de las medidas que debería impulsar el gobierno para facilitar la vida familiar. También indican que la crisis se evidencia en las posibilidades para tener hijos e hijas, puesto 
que un $89,5 \%$ afirma no tener descendencia por falta de medios económicos; una situación que - según la Memoria de la Situación Económica y Social de Galicia 2015 (CESG, 2016) - tiene su reflejo en el territorio gallego mediante su baja tasa de natalidad, puesto que desde el año 2008 el porcentaje de parejas con descendencia, sobre el total de hogares, ha ido disminuyendo de forma gradual (del $35,2 \%$ en el año 2008 al 33\% en el 2014).

Ante este panorama que pone de manifiesto los efectos de la crisis en el bienestar de las familias, profundizar en la percepción que éstas tienen acerca de su calidad de vida no es una cuestión baladí. En las últimas décadas, los estudios que indagaban en esta materia se centraban en la vida laboral y familiar, sin embargo, y de forma paulatina, han ido surgiendo investigaciones que sitúan sus análisis en la satisfacción de las personas con la conciliación de sus tiempos (Ford, Heinen y Langkamer, 2007; Valcour, 2007). Se entiende por satisfacción "una valoración cognitiva personal que realiza una persona de la vida y de los dominios de la misma, atendiendo a la calidad de su vida, a las expectativas y aspiraciones, a los objetivos conseguidos, basada en los propios criterios de la persona y que se realiza de forma favorable" (Clemente, Molero y González Sala, 2000: 189).

Bajo este enfoque, explicar la satisfacción vital desde una perspectiva amplia implica tomar en consideración la complacencia con las principales variables (internas y externas) de la vida cotidiana (trabajo, ingresos, familia, amistades, ocio, conciliación...). De este modo, cabe afirmar que la satisfacción en el ámbito laboral está relacionada con la referida al plano personal (Pérez-Pérez et al., 2015); al igual que tener problemas en el trabajo - o la ausencia del mismo - incide negativamente en la satisfacción en la esfera individual (Frey y Stutzer, 2005).

Desde este planteamiento, una falta de apoyo público en las políticas familiares afecta inexorablemente a la conciliación de las familias y, por ende, a su satisfacción y percepción de bienestar. Así lo evidencian los resultados derivados de la encuesta sobre la Calidad de Vida en el Trabajo desarrollada por el Ministerio de Trabajo e Inmigración (actual Ministerio de Empleo y Seguridad Social), que recogió por vez primera en el año 2010 una pregunta relativa a esta cuestión. El análisis realizado por Rico (2012) en torno a ella ha revelado que la satisfacción con la conciliación es más positiva cuando los trabajadores reciben ayudas sociales por parte de las empresas, cuando éstas facilitan la negociación colectiva y si se potencia el trabajo en equipo. Por el contrario, el nivel de satisfacción decae cuando el trabajador no alcanza los 40 años; cuanto mayor es el número de hijos/as menores de 15 años; si se cuenta con personas dependientes a cargo; cuanto mayor es el tamaño del municipio; cuando la formación es elevada; $y$, finalmente, al estar pluriempleado.

En este marco, las medidas de apoyo familiar - a nivel empresarial y social - son ineludibles para garantizar la satisfacción de los trabajadores y trabajadoras con la conciliación de sus tiempos personales, familiares y laborales. En consecuencia, se hace necesario dejar atrás políticas familiares orientadas a la reparación a posteriori de las carencias de las familias (deficit model), para desarrollar propuestas que pongan el acento en objetivos de carácter preventivo y de refuerzo de su autonomía (empowering-model), orientadas a: evitar la pérdida de capital humano (especialmente femenino); proteger el valor social que supone la infancia (el futuro 
colectivo trabajador del mañana); contribuir a que mujeres y hombres logren sus deseos de descendencia (el nivel de fecundidad actual se sitúa por debajo del deseado) y, por tanto, coadyuvar a paralizar el envejecimiento demográfico (González, 2011).

No obstante, lejos de caminar hacia prácticas de conciliación real, las tensiones para organizar los tiempos cotidianos continúan en aumento, especialmente para aquellas familias con hijos/as de corta edad. Situando el foco en el periodo de escolarización obligatoria, que en el contexto español comprende las etapas de Educación Primaria (de 6 a 12 años) y de Educación Secundaria Obligatoria (de 12 a 16 años), es durante la primera cuando las familias presentan mayores dificultades en la conciliación, lo que explica que los servicios escolares sean los más demandados para el cuidado de la infancia después de la ayuda de familiares (Caballo, Gradaílle y Merelas, 2012; Fraguela, Lorenzo y Varela, 2011; Sintes, 2015).

El presente trabajo tiene como objetivo indagar en el impacto que la crisis económica ha tenido en la organización de los tiempos de las familias con hijas e hijos que cursan Educación Primaria en la Comunidad Autónoma de Galicia, así como en las dificultades personales, familiares y laborales que éstas perciben para la conciliación. Se trata de un estudio que forma parte de una investigación más amplia (proyecto Concilia_d@s) ${ }^{1}$ orientada a conocer y analizar cómo organizan y concilian los tiempos cotidianos las familias gallegas del alumnado de esta etapa educativa, atendiendo a su lugar de residencia habitual (rural y urbano), a los tiempos escolares (calendarios y horarios lectivos) y a las actividades de ocio que realizan.

\section{Metodología}

Bajo las coordenadas científicas anteriormente presentadas, se desarrolla el proyecto de investigación Concilia_d@s, enmarcado en una línea de estudio en torno a los tiempos educativos y sociales que se ha venido desarrollando desde hace ya varias décadas y que tuvo su antecedente más inmediato en el proyecto Concilia (2008-2010), centrado en analizar la organización y conciliación de los tiempos cotidianos de la infancia perteneciente a familias que vivían en las ciudades gallegas. Una perspectiva de trabajo a la que se da continuidad en el proyecto Concilia_d@s, con un alcance más amplio, teniendo en consideración no solo el hábitat urbano sino también el contexto rural; sin obviar otras variables que inciden en la organización y conciliación de los tiempos, tales como el género, las condiciones laborales de los padres/madres, la formación recibida o los procesos de socialización.

La configuración del diseño de la muestra partió de un muestreo aleatorio simple adecuado a los criterios de proporcionalidad y representatividad, tomando

$1 \quad$ El artículo se inscribe en el proyecto de investigación "La Conciliación en los Tiempos Cotidianos de la Infancia en Galicia: Problemáticas Específicas y Alternativas Pedagógico-Sociales y Prácticas de Ocio en las Escuelas, Familias y Comunidades" (Concilia_d@s), financiado por la Consellería de Cultura, Educación y Ordenación Universitaria de la Xunta de Galicia en la convocatoria de Emergentes (2014-PG004), siendo investigadora principal la Profa. Dra. Rita Gradaílle Pernas. 
Datos de identificación de la muestra $(\%)$

\begin{tabular}{|c|c|c|c|c|}
\hline Provincia & $\begin{array}{l}\text { Contexto } \\
\text { territorial }\end{array}$ & $\begin{array}{l}\text { Titularidad } \\
\text { del centro }\end{array}$ & $\begin{array}{c}\text { Curso } \\
\text { de escolarización }\end{array}$ & $\begin{array}{c}\text { Persona } \\
\text { que responde }\end{array}$ \\
\hline $\begin{array}{c}\text { A Coruña }-27,5 \\
\text { Lugo - 19,1 } \\
\text { Ourense }-16,2 \\
\text { Pontevedra - } 37,2\end{array}$ & $\begin{array}{l}Z D P-46,6 \\
Z I P-18,9 \\
Z P P-34,6\end{array}$ & $\begin{array}{l}\text { Público - 72,3 } \\
\text { Privado - 27,7 }\end{array}$ & $\begin{array}{r}1 .^{\circ}-16,2 \\
2 .^{\circ}-17,1 \\
3 .^{\circ}-16,4 \\
4 .^{\circ}-16,2 \\
5 .^{\circ}-18,0 \\
6 .^{\circ}-16,0 \\
\text { ns } / \text { nc }-0,1\end{array}$ & $\begin{array}{c}\text { Madre }-78,1 \\
\text { Padre }-19,8 \\
\text { Madre y padre }-0,3 \\
\text { Otros }-0,3 \\
\text { ns } / \mathrm{nc}-1,4\end{array}$ \\
\hline
\end{tabular}

Nota: ZDP: zona densamente poblada. ZIP: zona intermedia poblada. ZPP: zona poco poblada.

como unidad de referencia los centros educativos. La conformación de la muestra de centros de Educación Primaria participantes en el estudio - cuyas características se recogen de forma resumida en la tabla 1 - se realizó en base a las siguientes variables de segmentación: provincia (A Coruña, Lugo, Ourense, Pontevedra); titularidad (público, privado); contexto territorial (zona densamente poblada, ZDP; zona intermedia, ZIP - alta, intermedia o baja - ; zona poco poblada, ZPP - alta,

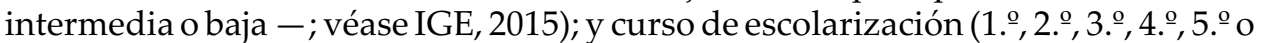
6.ํㅡㄹ Educación Primaria).

La población de referencia fue el alumnado gallego escolarizado en Educación Primaria en el curso académico 2014/2015, un total de 135.602 personas. La localización de los centros se hizo al azar, realizándose un contacto espontáneo y aleatorio, atendiendo siempre a la muestra final establecida. En los centros también se llevó a cabo aleatoriamente la asignación de grupos en los cursos de referencia; en total se entregaron 3400 cuestionarios, quedando la muestra configurada por 2037 encuestas válidas. Los cuestionarios fueron entregados en las aulas por integrantes del equipo investigador en el transcurso de la jornada lectiva, solicitándole al alumnado que se lo diera a sus familias -madre/tutora; padre/tutor; $u$ otra persona - y lo devolviera cumplimentado en el plazo de una semana. Se dejó a la libre elección de las familias quién respondía al cuestionario, siendo rellenado en un $78,1 \%$ de los casos por la madre/tutora y en el $19,8 \%$ por el padre/tutor (véase tabla 1).

Así, el principal instrumento de recogida de información del proyecto fue un cuestionario elaborado ad hoc para familias con hijas e hijos escolarizados en Educación Primaria en la Comunidad Autónoma de Galicia, abordando los siguientes bloques temáticos: datos personales; conciliación; tiempos compartidos y tiempos libres; recursos y servicios comunitarios; ocio, actividad física y salud. En concreto, los datos que se presentan toman como referencia el bloque temático denominado "conciliación" en el que se les pregunta a las familias acerca de la repercusión de la crisis en la conciliación de sus tiempos -mejoró, empeoró o no varió -, así como también se estudia su grado de acuerdo en una escala tipo Likert (donde $1=$ totalmente en desacuerdo y $4=$ totalmente de acuerdo) con diversas afirmaciones vinculadas a la armonización de los tiempos personales, familiares y laborales. 
El nivel de participación obtenido garantiza la representatividad de los resultados para el perfil de familias estudiado, que son extrapolables para el conjunto de familias gallegas con hijos e hijas escolarizados en esta etapa educativa, quedando fijado el nivel de error en el 2,2\% y el nivel de confianza en el 95,5\%. El análisis de los datos ha sido realizado con el programa informático IBM SPSS Statistics (versión 20.0 para Windows).

\section{Resultados}

A continuación, se exponen los principales resultados obtenidos en la investigación desarrollada, analizando cuál es la percepción de las familias participantes en el estudio respecto a la influencia de la situación de crisis en la conciliación. Así, se trata de conocer cuál es su grado de satisfacción sobre la organización de sus tiempos personales, familiares y profesionales, valorando si la crisis ha empeorado, mejorado o no ha variado la conciliación.

En lo tocante a la consideración que, en términos generales, tienen las familias encuestadas $(n=2037)$ sobre la incidencia de la situación de crisis en la conciliación de sus tiempos cotidianos (véase figura 1 ) algo más de la mitad de la muestra (53,7\%) señala que ésta no ha variado. A pesar de que puede tratarse de un dato positivo, pues evidencia que la situación de crisis no ha repercutido en la conciliación de los tiempos cotidianos, cabe tener en cuenta que un $39,6 \%$ de las personas participantes en el estudio afirma que ésta ha empeorado. No obstante, sí que existe una amplia diferencia respecto a las personas que consideran que con la crisis mejoró la armonización de los tiempos de su vida diaria; ya que solo un 6,7\% comparten esta opinión. Una situación que se deriva del efecto más impactante de la crisis: la falta de oportunidades de empleo, siendo la tasa de paro en Galicia del 16,5\% para los mayores de 25 años y del 42,5\% para los menores de esta edad (EPA, IV trimestre, 2015).

Respecto a los ítems "soy capaz de conseguir mis metas profesionales, familiares y personales de una forma satisfactoria" $\mathrm{y}$ "estoy satisfecho/a con mi organización de los tiempos de trabajo, para la familia y para mí mismo/a" (véase tabla 2), se observa que los datos porcentuales más elevados se encuentran en las opciones intermedias - "de acuerdo" y "en desacuerdo" - , situándose todas ellas entre un $34 \%$ y un $40 \%$ de la muestra. Por contra, el porcentaje de respuesta obtenido respecto a las alternativas "totalmente de acuerdo" y "totalmente en desacuerdo" se sitúa entre un $10 \%$ y un $16 \%$, respectivamente.

El hecho de que entre los porcentajes obtenidos los más elevados se encuentren en las opciones de respuesta "en desacuerdo" y "de acuerdo", sin apenas existir divergencias entre ellas (en ningún caso se superan los seis puntos porcentuales de diferencia), dificulta poder afirmar, de forma concisa y generalizada, cuál es la percepción de las familias.

Con todo, si se toma como referencia la agrupación de las opciones de respuesta en dos bloques: uno relativo a que no se comparten las afirmaciones planteadas, ya sea porque se está "totalmente en desacuerdo" o "en desacuerdo" y otro que da muestra de su conformidad ("totalmente de acuerdo" o "de acuerdo"), se 


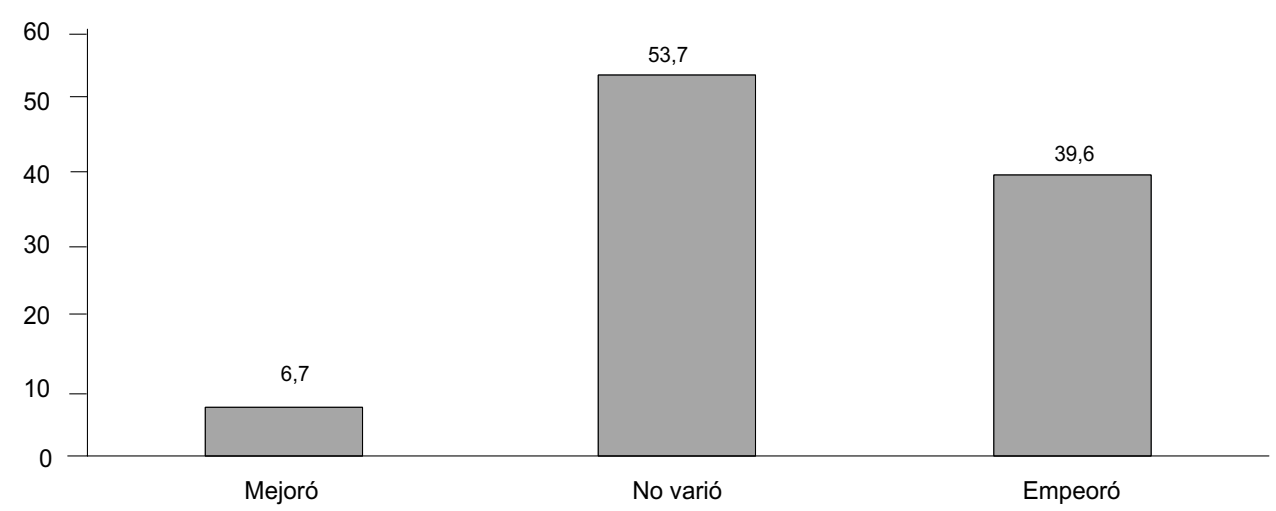

Figura 1 Percepción de la influencia de la situación de crisis en la conciliación (\%)

Tabla 2 Grado de acuerdo de las familias en relación con dos ítems vinculados con la satisfacción que se tiene con la conciliación de los tiempos cotidianos

\begin{tabular}{|c|c|c|c|c|c|}
\hline & & $\begin{array}{c}\text { Totalmente } \\
\text { en desacuerdo }\end{array}$ & En desacuerdo & De acuerdo & $\begin{array}{l}\text { Totalmente } \\
\text { de acuerdo }\end{array}$ \\
\hline $\begin{array}{l}\text { "Soy capaz de conseguir mis } \\
\text { metas profesionales, familiares }\end{array}$ & $f$ & 309 & 777 & 670 & 198 \\
\hline $\begin{array}{l}\text { y personales de una forma } \\
\text { satisfactoria" }\end{array}$ & $\%$ & 15,8 & 39,8 & 34,3 & 10,1 \\
\hline $\begin{array}{l}\text { "Estoy satisfecho/a con mi } \\
\text { organización de los tiempos }\end{array}$ & $f$ & 284 & 728 & 713 & 234 \\
\hline $\begin{array}{l}\text { de trabajo, para la familia y para } \\
\text { mí mismo/a" }\end{array}$ & $\%$ & 14,5 & 37,2 & 36,4 & 11,9 \\
\hline
\end{tabular}

observa que, a pesar de que la diferencia entre ambos bloques sigue siendo escasa, un mayor número de personas considera que no es capaz de conseguir sus metas profesionales, familiares y personales de una forma satisfactoria $(55,6 \%)$, a la vez que no están satisfechas con la organización de sus tiempos cotidianos (51,7\%). En este sentido, se constata que algo más de la mitad de las familias gallegas participantes en el estudio con hijos e hijas en Educación Primaria perciben malestar e insatisfacción respecto a la conciliación de los tiempos en su vida diaria.

Por otra parte, los resultados evidencian que existe una relación de dependencia significativa $\left(x^{2} 6=76,420 ; p<0,01\right)$ entre la incidencia de la crisis en la conciliación de los tiempos cotidianos y la capacidad de conseguir las metas profesionales, familiares y personales de una forma satisfactoria. Consecuentemente, es posible afirmar que las repercusiones de la crisis en la organización de los tiempos inciden en el grado de satisfacción de las familias acerca del logro de sus aspiraciones personales, familiares y laborales.

En concreto, se observa (véase tabla 3) que de las personas que piensan que la situación de crisis mejoró la distribución de los tiempos de su vida diaria $(\mathrm{n}=126)$, 
Tabla 3 Relación entre la incidencia de la crisis en la conciliación de los tiempos cotidianos y la capacidad de conseguir las metas profesionales, familiares y laborales de una forma satisfactoria

\begin{tabular}{|c|c|c|c|c|c|}
\hline & & \multicolumn{4}{|c|}{$\begin{array}{l}\text { Incidencia de la crisis en la conciliación de los tiempos } \\
\text { cotidianos }\end{array}$} \\
\hline & & & $\begin{array}{l}\text { Mejoró } \\
(n=126)\end{array}$ & $\begin{array}{l}\text { No varió } \\
(\mathrm{n}=1008)\end{array}$ & $\begin{array}{c}\text { Empeoró } \\
(\mathrm{n}=752)\end{array}$ \\
\hline \multirow{2}{*}{$\begin{array}{l}\text { "Soy capaz de conseguir mis } \\
\text { metas profesionales, familiares } \\
\text { y personales de una forma } \\
\text { satisfactoria" }\end{array}$} & Desacuerdo & $\begin{array}{l}\mathrm{F} \\
\%\end{array}$ & $\begin{array}{c}74 \\
58,8\end{array}$ & $\begin{array}{l}486 \\
48,2\end{array}$ & $\begin{array}{l}493 \\
65,5\end{array}$ \\
\hline & Acuerdo & $\begin{array}{l}\mathrm{F} \\
\%\end{array}$ & $\begin{array}{c}52 \\
41,2\end{array}$ & $\begin{array}{l}522 \\
51,8\end{array}$ & $\begin{array}{l}259 \\
34,4\end{array}$ \\
\hline
\end{tabular}

Nota: Estimación a partir de los datos porcentuales agrupados en dos bloques: "Desacuerdo" — totalmente en desacuerdo/en desacuerdo - y "Acuerdo" - totalmente de acuerdo/de acuerdo.

más de la mitad $(58,8 \%)$ señala que no es capaz de conseguir sus metas personales, familiares y profesionales satisfactoriamente. Unos resultados que evidencian que de entre quienes consideran que la conciliación ha progresado, cerca de un $60 \%$ no se siente con la competencia necesaria para lograr armonizar los diferentes ámbitos de su vida alcanzando mayores niveles de bienestar.

En la misma línea - pero con una divergencia porcentual mayor - se orienta la opinión de las personas que consideran que las dificultades para la conciliación de sus tiempos se han agravado como consecuencia de la crisis $(n=752)$. Así, de este conjunto, un $65,5 \%$ percibe que es incapaz de dar respuesta de un modo satisfactorio a las múltiples demandas a las que ha de hacer frente a nivel personal, familiar y laboral. Con todo, cabe destacar el $34,4 \%$ restante que, aun señalando que la conciliación de sus tiempos se ha deteriorado con la situación de crisis, indica que alcanza exitosamente sus objetivos.

Por el contrario, respecto a las familias que afirman que la conciliación no se ha alterado con motivo de la crisis $(n=1008)$, el hecho de que la diferencia entre quienes se ven capaces de conseguir sus metas satisfactoriamente $(51,8 \%)$ y aquellos que no lo hacen $(48,2 \%)$ sea de menos de cuatro puntos porcentuales, refleja que a pesar de ser en el único caso en el que algo más de la mitad de la muestra señale que es capaz de conciliar sus tiempos cotidianos de forma satisfactoria, la concordancia entre el grado de acuerdo no es tan clarificador como en las otras situaciones ("mejoró" - "empeoró").

En cuanto a la satisfacción de los progenitores con la organización de los tiempos de trabajo, para la familia y para sí mismos, se constata una relación de dependencia significativa $\left(x^{2} 6=89,265 ; p<0,01\right)$ entre dicha satisfacción y la percepción que tienen acerca de la incidencia de la crisis en la conciliación de sus tiempos cotidianos. De este modo, se confirma que su visión acerca del impacto de la crisis en la armonización de los tiempos cotidianos repercute en su sentimiento de satisfacción sobre esta cuestión.

En este sentido, tal y como se puede observar en la tabla 4, del conjunto de familias que aluden a que la conciliación se ha visto favorecida por la situación de crisis $(n=126)$, un $52,4 \%$ se siente conforme con la armonización de sus tiempos. Una 
Tabla 4 Relación entre la incidencia de la crisis en la conciliación y la satisfacción con la organización de los tiempos de trabajo, para la familia y para sí mismo

\begin{tabular}{|c|c|c|c|c|c|}
\hline & & \multicolumn{4}{|c|}{ Incidencia de la crisis en la conciliación de los tiempos cotidianos } \\
\hline & & & $\begin{array}{c}\text { Mejoró } \\
(n=126)\end{array}$ & $\begin{array}{l}\text { No varió } \\
(n=1012)\end{array}$ & $\begin{array}{c}\text { Empeoró } \\
(n=754)\end{array}$ \\
\hline \multirow{2}{*}{$\begin{array}{l}\text { "Estoy satisfecho/a con mi } \\
\text { organización de los tiempos } \\
\text { de trabajo, para la familia y } \\
\text { para mí mismo/a" }\end{array}$} & Desacuerdo & $\begin{array}{c}f \\
\%\end{array}$ & $\begin{array}{c}60 \\
47,6\end{array}$ & $\begin{array}{r}437 \\
43,2\end{array}$ & $\begin{array}{l}485 \\
64,3\end{array}$ \\
\hline & Acuerdo & $\begin{array}{c}f \\
\%\end{array}$ & $\begin{array}{c}66 \\
52,4\end{array}$ & $\begin{array}{l}575 \\
56,8\end{array}$ & $\begin{array}{l}269 \\
35,7\end{array}$ \\
\hline
\end{tabular}

Nota: Estimación a partir de los datos porcentuales agrupados en dos bloques: "Desacuerdo" — totalmente en desacuerdo/en desacuerdo - y "Acuerdo" — totalmente de acuerdo/de acuerdo.

perspectiva que refleja una mejora en la conciliación de los tiempos cotidianos, a la vez que un sentimiento de satisfacción con la organización que se realiza de los mismos; no pudiendo vincularse dicha percepción a las condiciones económicas que en líneas generales se manifiestan negativas - sino fundamentalmente a una mayor disponibilidad de tiempo por parte de los adultos.

Además, de entre las personas que apuntan que en su caso la conciliación es la misma con independencia de la situación de crisis $(n=1012)$, el porcentaje de quienes están satisfechos con la organización de su vida diaria $(56,8 \%)$ es mayor que el de aquellos que no lo están.

Una perspectiva diferente, sobre este bienestar con la sincronización de los tiempos cotidianos, es la de quienes consideran que su conciliación se ha visto afectada negativamente por la crisis $(n=754)$; ya que un $64,3 \%$ señala no estar satisfecho/a con la organización de su vida diaria. Se trata de una visión negativa sobre la temática de estudio, en tanto que evidencia un incremento de las dificultades en la conciliación de su vida cotidiana fruto de la recesión económica, a la vez que un sentimiento de malestar respecto a la misma. Frente a este dato, cerca de un $36 \%$ - aun percibiendo que la conciliación empeoró con motivo de la crisis ? se siente satisfecho respecto a la armonización de sus tiempos cotidianos.

Con todo, cabe mencionar que la diferencia porcentual entre quienes consideran que "mejoró" $(\mathrm{n}=126)$, "no varió" $(\mathrm{n}=1012)$ o "empeoró" $(\mathrm{n}=754)$ la conciliación respecto al grado de acuerdo ("desacuerdo" - "acuerdo") con este ítem ("Estoy satisfecho/a con mi organización de los tiempos de trabajo, para la familia y para mí mismo/a"), se incrementa progresivamente en función de la percepción más o menos positiva que se tenga sobre la influencia de la crisis en la conciliación. En concreto, del conjunto de personas que señalan que se enriqueció la organización de su vida diaria, existe una diferencia de aproximadamente cinco puntos porcentuales entre aquellas que están satisfechas con la organización de sus tiempos y las que no lo están; una cifra que aumenta en el caso de quienes afirman que no hubo variaciones en la conciliación, y todavía más entre los progenitores que perciben un deterioro en la conciliación debido a la situación de crisis.

Estas divergencias evidencian que entre las familias para las que la conciliación ha mejorado o no se ha modificado, el porcentaje total de las que perciben 
bienestar y de las que no lo hacen es semejante; mientras que en el caso de las personas que indican que las dificultades para la organización de sus tiempos se agravaron con la crisis la percepción de insatisfacción es predominante.

\section{Discusión y conclusiones}

Los resultados obtenidos constatan que la crisis económica ha impactado en el bienestar de las familias gallegas del alumnado de Educación Primaria, tanto en lo referido a su grado de satisfacción respecto a las posibilidades de las que disponen para la consecución de sus metas profesionales, familiares y personales, como acerca de la organización de sus tiempos cotidianos. Pues más de la mitad de las personas participantes en el estudio perciben malestar e insatisfacción respecto a la conciliación.

Estos datos coinciden con la tendencia señalada en el informe "Crisis Económica, Calidad del Trabajo e Integración Social" (Gallie, 2013) en el que, analizando el impacto de la crisis económica, se refleja que entre el año 2004 y el 2010 se produjo un incremento significativo del conflicto existente en la armonización de la vida laboral y familiar en la mayoría de los países europeos. Según dicho estudio, son varios los factores que contribuyeron a la existencia de esta realidad, entre ellos: el incremento de la desregulación horaria, la ampliación del tiempo de dedicación al trabajo y el aumento de la inseguridad laboral. No obstante, el factor más decisivo es la elevación de la tasa de desempleo, al que? según datos de la Fundación Pfizer (2014) ? se añaden otros como la disminución de ingresos. En consecuencia, cabe afirmar que quienes tienen mayores dificultades para abordar las obligaciones familiares y disponen de menos tiempo personal muestran un menor nivel de satisfacción con la organización de sus tiempos (Böhnke, 2005).

De forma específica, en lo referido al grado de satisfacción de las familias gallegas con la armonización de sus tiempos cotidianos y a la capacidad de logro de sus metas, los hallazgos del estudio ponen de manifiesto que algo más de la mitad de la muestra percibe malestar e insatisfacción en la conciliación de las diversas temporalidades. Así, un 55,6\% considera que no es capaz de conseguir sus metas profesionales, familiares y personales de una forma satisfactoria y un $51,7 \%$ afirma no estar satisfecho/a con la organización de sus tiempos cotidianos.

Unos resultados que concuerdan con los ofrecidos por la "Encuesta sobre la Conciliación de la Vida Laboral, Familiar y Personal" (2010) llevada a cabo por el Instituto Vasco de Estadística (Eustat) sobre la población activa de Euskadi, en la cual se evidenció que el $25,9 \%$ de la muestra tenía dificultades altas para alcanzar las metas relacionadas con su ámbito familiar y personal y un $21,9 \%$ una dificultad media. Es decir, cerca de la mitad de los participantes $(47,8 \%)$ indicaron tener problemas altos o moderados para lograr sus objetivos familiares y personales, al tiempo que un $42,4 \%$ señalaba una satisfacción media-baja con la conciliación. En esta línea, diversas investigaciones atestiguan que, generalmente, las personas que tienen mayores dificultades para armonizar sus tiempos cotidianos muestran un menor grado de satisfacción con su vida (Valcour, 2007; Carmon, Miller y Brasher, 2013). 
Aun sin existir grandes diferencias porcentuales, de la investigación desarrollada se desprende que algo más de la mitad de las familias encuestadas consideran que la conciliación de sus tiempos cotidianos no varió con motivo de la situación de crisis. Sin embargo, esta afirmación no implica que todas ellas tengan un elevado grado de satisfacción con la conciliación, ya que predominan las que reconocen que no son capaces de alcanzar sus metas personales, familiares y profesionales satisfactoriamente, así como aquellas que muestran insatisfacción con la organización de los tiempos de su vida diaria. Merece ser destacado que casi un $40 \%$ de la muestra percibe que la armonización de sus tiempos se ha visto mermada como consecuencia de la crisis; un dato que refleja que, en lo referido a la conciliación, el bienestar y la calidad de vida, las familias gallegas han resultado intensamente perjudicadas por la recesión económica de los últimos años.

En consecuencia, los resultados reafirman la necesidad de emprender un proceso de cambio orientado a la mejora de la conciliación de los padres y madres con hijos e hijas escolarizados en la etapa de Educación Primara (6-12 años), constituyendo la corresponsabilidad familiar un eje esencial. Así, lo importante no es solo alcanzar una distribución igualitaria de las acciones a desarrollar, en cantidad y calidad, sino la satisfacción de todas las personas; de ahí que el diálogo y el consenso sean dos elementos esenciales para promover un cambio de perspectiva (Maganto y Etxeberria, 2012). También el ámbito escolar incide directamente en la organización de los tiempos de las familias, percibiéndose conflictos entre los ritmos escolares y las jornadas laborales de los progenitores. Esta compleja situación de "encaje" de tiempos, a la que cotidianamente deben hacer frente las familias, exige que las transformaciones que se lleven a cabo respecto a la organización y distribución temporal se deban enfocar desde una perspectiva social y educativa (Varela, Varela y Lorenzo, 2016).

Sin embargo, a pesar de esta realidad de dificultades en la conciliación, no existe un compromiso suficiente por parte del Estado y de los poderes públicos a la hora de ofrecer a las familias los servicios necesarios y las facilidades adecuadas para que puedan desarrollar la cotidianeidad de su vida de forma óptima (Monteiro y Domingos, 2013). Una circunstancia que se agrava si se tiene en cuenta que siguen siendo las mujeres las que soportan principalmente las responsabilidades familiares (Alcañiz, 2013), viendo así reducidas sus oportunidades de desarrollo en todos los ámbitos de su vida. De facto, tal y como indicamos con anterioridad (véase tabla 1), en el estudio que se presenta las familias eligieron libremente quién de sus miembros daba respuesta al cuestionario, siendo contestado mayoritariamente por las madres $(78,1 \%)$.

En aras de promover la igualdad de oportunidades entre ambos sexos, las directrices de las políticas sociales se han de orientar a garantizar que tanto mujeres como hombres puedan desarrollar sus compromisos laborales y familiares, sin que esta doble responsabilidad mengue sus derechos sociales (Gornick y Meyers, 2009), ni la posibilidad de alcanzar un equilibrio saludable entre las múltiples obligaciones a las que cotidianamente han de dar respuesta (Darthy y McCarthy, 2007); favoreciendo así el desarrollo armónico e integral de la infancia. De ahí que, ante la 
crisis económica actual que repercute en el bienestar de las familias, la conciliación - más allá de ser entendida como un asunto de carácter privado - deba integrar cuestiones referidas a la igualdad de oportunidades entre mujeres y hombres, a la mejora de las condiciones laborales y a la asunción de compromisos colectivos (administración pública, sector empresarial, instituciones educativas, etc.) orientados al desarrollo de cambios estructurales en la organización de los tiempos cotidianos socialmente vigente.

\section{Referencias bibliográficas}

Alcañiz, Mercedes (2013), “Estrategias de conciliación y segmentación social: la doble desigualdad", Sociologia, Problemas e Práticas, 73, pp. 33-57.

Andrade, Luis Gerardo, y René Landero (2015), “Bases teóricas del conflicto trabajo-familia", Revista de Psicología (Universidad de Antioquía), 7 (1), pp. 185-198.

Antón, Antonio (2012), “Política social en tiempos de crisis", Cuadernos de Trabajo Social, 25 (1), pp. 49-62.

Baxter, Janeen, y Jenny Chesters (2011), “Perceptions of work-family balance: how effective are family-friendly policies?", Australian Journal of Labour Economics, 14 (2), pp. 139-151.

Böhnke, Petra (2005), First European Quality of Life Survey. Life Satisfaction, Happiness and Sense of Belonging, Luxemburgo, Office for Official Publications of the European Communities.

Borràs, Vicent, Teresa Torns, y Sara Moreno (2007), “Las políticas de conciliación: políticas laborales versus políticas de tiempo", Papers, 83, pp. 83-96.

Burke, Ronald (2009), “Working to live or living to work: should individuals and organizations care?", Journal of Business Ethics, 84 (2), pp. 167-172.

Caballo, Maㅡㄹén, Rita Gradaílle, y Tania Merelas (2012), “Servicios socioeducativos y corresponsabilidad en la conciliación de los tiempos familiares: situación de la infancia en la Galicia urbana", Pedagogía Social - Revista Interuniversitaria, 20, pp. 179-202.

Carmon, Anna, Amy Miller, y Kristen Brasher (2013), “Privacy orientations: a look at family satisfaction, job satisfaction and work-life balance", Communication Reports, 26 (2), pp. 101-112.

Castro, Teresa, y Marta Seiz (2014), “La transformación de las familias en España desde una perspectiva sociodemográfica", en Fundación Foessa (org.), VII Informe sobre Exclusión y Desarrollo Social en España (documento de trabajo 1.1), Madrid, Fundación Foessa / Cáritas, pp. 31-36, recuperado de: http://www.foessa2014.es/informe/uploaded/documentos_trabajo/13112014045006_ 7884.pdf (última consulta en junio 2016).

CIS - Centro de Investigaciones Sociológicas (2014), Opiniones y Actitudes sobre la Familia (II), estudio n. ${ }^{\circ}$ 3032, julio 2014, Madrid, CIS.

Clemente, Antonio, Rosa Molero, y Francisco González Sala (2000), “Estudio de la satisfacción personal según la edad de las personas", Anales de Psicología, 16 (2), pp. 189-198. 
CESG - Consello Económico e Social de Galicia (2016), Memoria sobre a Situación Económica e Social de Galicia, 2015, Xunta de Galicia, recuperado de: http://www.ces-galicia.org/publicacions/memoria-socioecon\%C3\%B3mica/memori a-sobre-situaci\%C3\%B3n-econ\%C3\%B3mica-e-social-galicia-2015 (última consulta en junio 2016).

Darthy, Colette, y Alma McCarthy (2007), “Work-family conflict: an exploration of the differential effects of a dependent child's age on working parents", Journal of European Industrial Training, 31 (7), pp. 530-554.

ECVT (2010), Encuesta de Calidad de Vida en el Trabajo, Madrid, Ministerio de Trabajo e Inmigración.

Eustat (2010), Encuesta sobre la Conciliación de la Vida Laboral, Familiar y Personal, recuperado de:

http://www.eustat.eus/document/datos/cuestionarios/cues_cvl10_c.pdf (última consulta en junio 2016).

Ford, Michael, Beth Heinen, y Krista Langkamer (2007), “Work and family satisfaction and conflict: a meta-analysis of cross-domain relations", Journal of Applied Psychology, 92 (1), pp. 57-80.

Fraguela, Raúl, Juan José Lorenzo, y Lara Varela (2011), “Escuela, familias y ocio en la conciliación de los tiempos cotidianos de la infancia", Revista de Investigación Educativa, 29 (2), pp. 429-446.

Fraguela, Raúl, Juan José Lorenzo, Tania Merelas, y Lara Varela (2013), “Tiempos escolares y conciliación: análisis de familias con hijos en educación secundaria obligatoria (12-16 años)", Revista de Investigación Educativa, 31 (2), pp. 431-446.

Frey, Bruno, y Alois Stutzer (2005), "Happiness research: state and prospects", Review of Social Economy, 62 (2), pp. 207-228.

Fundación Pfizer (2014), Estudio sobre Conciliación y Familia. Informe de Resultados, recuperado de: https://www.fundacionpfizer.org/sites/default/files/pdf/informe_ final_estudio_foro_debate_social_2014.pdf (última consulta en junio 2016).

Gallie, Duncan (org.) (2013), Crisis Económica, Calidad del Trabajo e Integración Social. Principales Resultados de la 2. ${ }^{\underline{a}}$ y 5. ${ }^{\underline{a}}$ Ediciones de la Encuesta Social Europea, Oxford, Oxford University Press.

González, María José (2011), “Políticas de familia e infancia, una inversión social para tiempos de bonanza y crisis económica", en Federación de Cajas de Ahorros Vasco-Navarras (org.), El Estado de Bienestar en la Encrucijada. Nuevos Retos ante la Crisis Global, Vitoria-Gasteiz, Federación de Cajas de Ahorros Vasco-Navarras, pp. 250-260.

Gornick, Janet, y Marcia Meyers (2009), “Institutions that support gender equality in parenthood and employment transforming family divisions of labor", en Janet C. Gornick y Marcia K. Meyers (orgs.), Gender Equality. Transforming Family Divisions of Labor, Londres, Verso, pp. 3-64.

IGE - Instituto Galego de Estatística (2015), Panorama Rural-Urbano, Santiago de Compostela, Instituto Galego de Estatística, recuperado de: http://www.ige.eu/web/mostrar_seccion.jsp?idioma=gl\&codigo=0701 (última consulta en junio 2016).

Maganto, Juana María, y Amaia Etxeberria (2012), “La corresponsabilidad como base de la conciliación", en Juana María Maganto (org.), Programa Concilia. Concilia en Tu 
Vida. La Conciliación de la Vida Personal, Familiar y Académico-Laboral en Adolescentes, Jóvenes y Adultos, Madrid, Ediciones Pirámide, pp. 163-216.

Ministerio de Sanidad, Servicios Sociales e Igualdad (2015), Plan Integral de Apoyo a la Familia (2015-2017), recuperado de: http://www.msssi.gob.es/ novedades/docs/PIAF-2015-2017.pdf (última consulta en junio 2016).

Monteiro, Rosa, y Liliana Domingos (2013), “O sentido do direito à conciliaçao: vida profissional, familiar e pessoal numa autarquia", Sociologia, Problemas e Práticas, 73, pp. 59-77.

Pérez-Pérez, Manuela, María José Vela-Jiménez, Silvia Abella-Garcés, y Ángel Martínez-Sánchez (2015), “El enriquecimiento trabajo-familia: nuevo enfoque en el estudio de la conciliación y la satisfacción laboral de los empleados", Universia Business Review, 45, pp. 16-33.

Rico, Paz (2012), “Satisfacción con la conciliación laboral y familiar de los asalariados en España", Revista de Economía Laboral, 9, pp. 30-45.

Sánchez-Vidal, María Eugenia, David Cegarra-Leiva, y Juan Gabriel Cegarra-Navarro (2011), “influye el conflicto trabajo-vida personal de los empleados en la empresa?", Universia Business Review, 29, pp. 100-115.

Sintes, Elena (2015), Escola a Temps Complet. Cap a Un Model d'Educació Compartida, Barcelona, Fundació Jaume Bofill.

Valcour, Monique (2007), “Work-based resources as moderators of the relationship between work hours and satisfaction with work-family balance", Journal of Applied Psychology, 92 (6), pp. 1512-1523.

Varela, Laura, Lara Varela, y Juan José Lorenzo (2016), “La escuela y la conciliación de los tiempos de las familias del alumnado de Educación Primaria en Galicia", Bordón, 68 (4), pp. 179-194.

Rubén Martínez García. Diplomado en Magisterio de Educación Primaria y licenciado en Psicopedagogía. Contratado predoctoral FPU (Subprograma Nacional de Formación de Profesorado Universitario, Ministerio de Educación, Cultura y Deporte) en el grupo de investigación de Pedagogía Social y Educación Ambiental (SEPA interea) de la Universidad de Santiago de Compostela (España). Facultad de Ciencias de la Educación (Campus Vida), Rúa Prof. Vicente Fráiz Andón, s/n, 15782, Santiago de Compostela, A Coruña (Galicia-España). Teléfono: 881813753. E-mail: ruben.martinez.garcia@usc.es

Ángela L. de Valenzuela. Diplomada y habilitada al grado de Educación Social. Contratada predoctoral FPI (Formación de Personal Investigador, Ministerio de Economía y Competitividad) en el grupo de investigación de Pedagogía Social y Educación Ambiental (SEPA interea) de la Universidad de Santiago de Compostela (España). Facultad de Ciencias de la Educación (Campus Vida), Rúa Prof. Vicente Fráiz Andón, s/n, 15782, Santiago de Compostela, A Coruña (Galicia-España). Teléfono: 881813753. E-mail: angela.devalenzuela@usc.es 
Laura Varela Crespo. Doctora en Ciencias de la Educación. Profesora ayudante doctora en el Departamento de Pedagogía y Didáctica de la Universidad de Santiago de Compostela y miembro del grupo de investigación de Pedagogía Social y Educación Ambiental (SEPA interea) de la Universidad de Santiago de Compostela (España). Facultad de Ciencias de la Educación (Campus Vida), Rúa Prof. Vicente Fráiz Andón, s/n, 15782, Santiago de Compostela, A Coruña

(Galicia-España). Teléfono: 881813748. E-mail: laura.varela@usc.es

Receção: 11 de junho de 2016 Aprovação: 15 de julho de 2017 